\title{
11 „Verlorene“ Rachentamponade
}

Berthold Bein, Elmar Biermann

\subsection{Klinischer Fall}

Während einer OP im Bereich der Nasennebenhöhlen wird eine Rachentamponade eingelegt. Eine Dokumentation der Rachentamponade auf dem Narkoseprotokoll erfolgt nicht. Beim Ankleben der sterilen Abdeckung ist dem Operateur das Rückholbändchen im Weg und wird von ihm abgeschnitten, ohne dies klar zu kommunizieren. Bei Narkoseausleitung deutet äußerlich nichts mehr auf die eingelegte Rachentamponade hin, die jetzt auch dem betreuenden Anästhesisten nicht mehr bewusst ist.

Nach der Extubation zeigt sich das Bild eines Laryngospasmus, welcher gemäß des klinikeigenen Algorithmus mittels Narkosevertiefung und Maskenbeatmung therapiert wird, bis das auslösende Agens plötzlich wieder ins Bewusstsein des Anästhesisten dringt. Daraufhin problemlose Entfernung der in den Larynxeingang prolabierten Tamponade.

\subsection{Konsequenzen für den Patienten}

Aufgrund der Atemwegsobstruktion war nur eingeschränkte Ventilation möglich. Allerdings fiel die pulsoxymetrisch gemessene Sättigung nie unter $90 \%$ ab. Insofern keine bleibende Beeinträchtigung.

\subsection{Interpretation aus Sicht des Anästhesisten}

Bei einer Operation im Mund-Rachen-Bereich wird eine Rachentamponade eingelegt. Der Operateur schneidet das Rückholbändchen ab, die Rachentamponade verbleibt auch über die Extubation hinweg und verursacht einen Laryngospasmus. Noch während des Behandlungsversuchs wird die auslösende Ursache wieder erinnert und beseitigt. Der
Patient ist zwar gefährdet, kommt aber nicht zu Schaden. Dem Bericht ist schließlich noch zu entnehmen, dass eine Dokumentation der Rachentamponade auf dem Narkoseprotokoll nicht erfolgte.

Medizinisch ist zu dem Fall wenig zu sagen: Der Laryngospasmus wurde korrekt erkannt und nach einem Standard-Algorithmus mit initialer Vertiefung der Anästhesie und Maskenbeatmung korrekt und offensichtlich innerhalb voll tolerabler Oxygenierungsgrenzen behandelt. Umso interessanter ist die Analyse des Falles unter den Human-Factor-Gesichtspunkten „Teamarbeit“ und „Kommunikation mit dem Operateur“" und dem Aspekt einer sorgfältigen Dokumentation. Bei derartigen Operationen kann es zweifellos jederzeit vorkommen, dass der Operateur ein Rückholbändchen durchschneidet (z.B. versehentlich oder weil es im OP-Gebiet stört etc.). Es stellt sich dann aber die Frage, wie die weitere Entwicklung der geschilderten Problemsituation vermieden werden kann?

1. Das Ereignis sollte unmissverständlich kommuniziert werden. Dies ist im vorliegenden Fall nicht geschehen.

2. Operateur und Anästhesist sollten sich auf ein Vorgehen einigen, wer von beiden am Ende der Operation die Rachentamponade entfernt. Durch ein standardisiertes Vorgehen bleibt die Tatsache gut in Erinnerung und die Wahrscheinlichkeit des Vergessens sinkt.

3. Schließlich sollte der Anästhesist die Tatsache, dass eine Rachentamponade eingelegt wurde, auch auf dem Narkoseprotokoll dokumentieren. Dies dient insbesondere auch dazu, dass bei unerwarteter Ablösung ein nachfolgender Anästhesist während der laufenden Prozedur vollen Zugriff auf alle notwendigen Informationen hat. 


\subsection{Analyse aus Sicht des Juristen}

Es darf davon ausgegangen werden, dass es um eine HNO-Operation ging, bei der routinemäßig eine Rachentamponade einlegt wird. Weiter darf unterstellt werden, dass dem Operateur bekannt ist, dass der Patient bei noch liegender Rachentamponade Probleme bei der Extubation haben wird. Nach den in der interdisziplinären Kooperation geltenden Grundsätzen der strikten Arbeitsteilung, ergänzt durch den Vertrauensgrundsatz, ist zunächst jeder Fachvertreter nur für die seinem Fachgebiet - nach dem Inhalt der Weiterbildungsordnung und/oder konkreten Absprachen vor Ort zukommenden Leistungen zuständig und verantwortlich. Dürfen diese Grundsätze auch so verstanden werden, dass der Operateur sich zurücklehnen darf, wenn er das seinige getan hat und keine Rücksicht darauf nehmen muss, welche Bedeutung seine Maßnahmen für den Partner in der Behandlung, den Anästhesisten hat? Oder sind die beiden Grundsätze vielmehr zu ergänzen durch die Verpflichtung zur Kommunikation und Kooperation, zur Abstimmung der Maßnahmen?

Dazu hat der Bundesgerichtshof in einem Urteil vom 26.02.1991 angesichts einer Schieloperation (BGH, MedR 1999, 321) Stellung genommen. Der BGH führt aus:

„Zwar hat der erkennende Senat ... den Grundsatz aufgestellt, dass jeder Arzt denjenigen Gefahren zu begegnen hat, die in seinem Aufgabenbereich entstehen, und dass er sich, so lange keine offensichtlichen Qualifikationsmängel oder Fehlleistungen erkennbar werden, darauf verlassen darf, dass auch der Kollege des anderen Fachgebietes seine Aufgaben mit der gebotenen Sorgfalt erfüllt, ohne dass insoweit eine gegenseitige Überwachungspflicht besteht. Hieraus kann jedoch nicht abgeleitet werden, dass eine $A b-$ stimmung zwischen mehreren an einer Heilmaßnahme beteiligten Ärzten - hier: Anästhesist und Operateur - in solchen Fällen unterlassen werden dürfe, in denen sich die Gefährdung des Patienten gerade aus dem Zusammenwirken mehrerer Ärzte bzw. einer Unvereinbarkeit der von ihnen angewendeten Methoden oder Instrumente ergibt. Der dargestellte Vertrauensgrundsatz kann nämlich nur in solchen Konstellationen gelten, in denen es um Gefahren geht, die ausschließlich dem Aufgabenbereich eines der betei- ligten Ärzte zugeordnet sind, während im Streitfall die Schädigung der Klägerin gerade daraus entstanden ist, dass die von den beteiligten Ärzten angewendeten Maßnahmen für sich genommen jeweils beanstandungsfrei waren und das besondere Risiko sich erst aus der Kombination der beidseitigen Maßnahmen ergeben hat. ... Aus dem hierfür aufgestellten Vertrauensgrundsatz kann jedoch nicht hergeleitet werden, dass unter den Umständen des Streitfalls eine Abstimmung zwischen Anästhesist und Operateur über die Wahl der Narkosemethode bzw. die bei der Operation zum Einsatz gelangenden Instrumente entbehrlich gewesen wäre. Insbesondere vermag jener Grundsatz die Pflichten der beteiligten Ärzte gegenüber dem Patienten nicht in solchen Fällen zu begrenzen, in denen sich wie im Streitfall das besondere Risiko der Heilmaßnahme gerade aus dem Zusammenwirken zweier verschiedener Fachrichtungen und einer Unverträglichkeit der von ihnen verwendeten Methoden oder Instrumente ergibt. Der erkennende Senat hat ... ausgeführt, dass bei Beteiligung mehrerer Ärzte eine gegenseitige Informationspflicht zu den Schutzpflichten dem Patienten gegenüber gehöre. Schon hiernach liegt eine Verpflichtung der beteiligten Ärzte zur Abstimmung über die Vereinbarkeit von Narkose- und Operationsmethode auf der Hand. ... Da ... das Wohl des Patienten oberstes Gebot und Richtschnur ist, muss für diese Zusammenarbeit der Grundsatz gelten, dass die beteiligten Ärzte den spezifischen Gefahren der Arbeitsteilung entgegenwirken müssen und es deshalb bei Beteiligung mehrerer Ärzte einer Koordination der beabsichtigten Maßnahmen bedarf, um zum Schutz des Patienten einer etwaigen Unverträglichkeit verschiedener von den Fachrichtungen eingesetzten Methoden oder Instrumente vorzubeugen. Unter diesem Blickpunkt ist auch dann, wenn insoweit keine ausdrückliche Vereinbarung zwischen den beteiligten Fachrichtungen besteht, schon nach allgemeinen Grundsätzen eine Pflicht der beteiligten Ärzte zu bejahen, durch hinreichende gegenseitige Information und Abstimmung vermeidbare Risiken für den Patienten auszuschließen.“

Vor dem Hintergrund des Primats der Sicherheit des Patienten verlangt die Rechtsprechung also, dass die Fachvertreter auch „über den Tellerrand hinausschauen" und prüfen, ob und inwieweit die von ihnen eingesetzten Medikamente und/oder Verfahren kompatibel sind. Selbst wenn das Rückholbändchen versehentlich abgeschnitten worden wäre, wird man den Operateur für verpflichtet hal- 
ten müssen, am Ende der OP diese Feststellung zu treffen und dies zu kommunizieren. Wäre der Patient geschädigt worden, spricht viel dafür, dass der Operateur die rechtliche Verantwortung für diesen Zwischenfall trägt.

\subsection{Weiterführende Gedanken}

Die Zusammenarbeit im OP erfordert unabhängig von der geschilderten Situation ein hohes Maß an Kommunikation und Abstimmung. Diese verläuft meist abhängig von den beteiligten Personen mehr oder weniger effizient. Eine gute Möglichkeit, diese Kommunikation im Hinblick auf sicherheitsrelevante Maßnahmen zu formalisieren, stellt die WHO-Checkliste dar ( $\vee$ Abb. 23.1), die präoperativ vor Narkoseeinleitung („Sign In“), vor Beginn der chirurgischen Maßnahmen („Team Time Out“) und unmittelbar nach Beendigung der chirurgischen Maßnahmen („Sign Out“) standardisiert wichtige Informationen abfragt (http://www.who.int/ patientsafety/safesurgery/ss_checklist/en/index. html).

\section{Take Home Message}

Operateur und Anästhesist müssen über alle relevanten Maßnahmen miteinander kommunizieren. Eine formalisierte Kommunikation in Form der WHO-Checkliste ist in diesem Zusammenhang empfehlenswert. 